\title{
SACRA ETHNOGEOGRAPHICA CELTICA. MYTHOS, EPOS E RELIGIO NELLE FONTI CLASSICHE E TARDOANTICHE DELLA BRITANNIA E DELLIRLANDA
}

\author{
Fausto Iannello \\ Università degli Studi di Messina \\ faustoiannello@gmail.com
}

\begin{abstract}
RESUMEN
El objetivo de este artículo es mostrar, a través de la clasificación, comparación y análisis de textos específicos clásicos y tardoantiguos griegos y latinos, cómo las fuentes antiguas sobre Britania e Irlanda están, tanto directa como indirectamente, basadas principalmente en datos e información histórico-religiosa, según la expresión de un punto de vista historiográfico que, en sus raíces, pretendía reunir y verbalizar el marco épico y mítico de los celtas insulares. Además, esas fuentes tienen un interés historiográfico, geográfico y etnográfico, que se expresa en mayor detalle en la adquisición de topónimos y etnónimos que no omiten elementos sagrados y religiosos, por lo que el conjunto de fuentes reunidas persiste como antes, como testimonio real de un mundo religioso que, desde el punto de vista clásico, nutre la fascinación geo-historiográfica y mitopoyética.

Palabras Clave: Celtas insulares, Britania e Irlanda en la Antigüedad Tardía y Alta Edad Media, fuentes griegas y latinas de celtas insulares, Pictos, Escotos, religión celta, mitología celta, geografía clásica, historiografía griega y latina.
\end{abstract}

\author{
SACRA ETHONOGEOGRAPHICA CELTICA. MYTHOS, EPOS AND RELIGION \\ IN CLASSICAL AND LATE ANTIQUE SOURCES OF BRITANNIA AND IRELAND
}

\section{AbSTRACT}

Aim of this article is to show, through the classification, comparison and analysis of Greek and Latin specific classical and late antique texts, how the ancient sources on Britannia and Ireland are, both directly and indirectly, mainly based on historico-religious information and data, as the expression of a historiographic point of view that, back to its roots, was aimed to collect and convey the mythical and epic framework of insular Celts. Moreover, those sources show a historiographic, geographic and etnographic interest, which is expressed in greater detail in the acquisition of toponyms and etnonyms that does not overlook sacred and religious elements, so that the ensemble of the collected sources persists as before, as a real testimony of a religious world that, from the classical point of view, fosters both a geo-historiographic and mythopoietic allure.

KeYwords: Insular Celts, Britain and Ireland in Late Antiquity and Early Middle Ages, Greek and Latin Sources of Insular Celts, Picts, Scots, Celtic Religion, Celtic Mythology, Classical Geography, Greek and Latin Historiography. 


\section{PREMESSA}

Attraverso un lavoro di classificazione, comparazione e analisi di specifici testi greci e latini di età classica e tardoantica, nel presente contributo si cercherà di dimostrare come la visione comune che si aveva sulla Britannia e l'Irlanda nelle età in oggetto fosse principalmente radicata su notizie e dati di ambito storico-religioso veicolati da una tendenza storiografica che, in prima istanza, si proponeva di ricostruire e tramandare il quadro mitico ed epico del Celti insulari. Inoltre, le fonti che saranno qui offerte attestano un interesse storiografico, geografico ed etnografico che si esprime dettagliatamente in una capillare acquisizione di toponimi e di etnonimi che, già a partire dal loro etimo, permarrebbero oscuri qualora non fossero ricondotti a precisi elementi sacrali, quindi religiosi, al punto che l'insieme delle fonti qui raccolte giungono a costituire la prima e imprescindibile testimonianza scritta di un mondo religioso che, nella visione già classica, alimentava un interesse e delle riletture tanto etnografiche e geo-storiografiche quanto simboliche e mitopoietiche.

\section{BRITANNIA}

È noto che la Britannia dei secoli V e VI può storicamente classificarsi come "Sub-Roman Britain" o "Age of Arthur", qualificazioni con le quali si tende a individuare e rappresentare un arco di tempo contrassegnato da una commistione culturale alquanto esclusiva, fondata, infatti, su una straordinaria sintesi di elementi romani, celtici e cristiani, e priva di qualsivoglia riferimento a una sostanziale e concreta decadenza di quelle terre. Tale periodo "post-romano" avrà termine con la definitiva espansione degli Anglosassoni nell'occidente dell'isola e, in particolare, negli anni tra il 597 e il 604, con l'evangelizzazione del Kent da parte di Agostino di Canterbury².

Di norma, negli scritti degli autori latini quello di Britannia è, sì, un toponimo concettualmente concepito e utilizzato in modo polivalente ${ }^{3}$, ma per loro la Britannia è l'altrove, un essere al di là, un alter orbis, insomma, per usare le parole di Velleio Patercolo 4 , sì che i suoi popoli, abitualmente noti come Britanni, sono detti vivere "del tutto fuori del mondo»: At nos hinc alii sitientis ibimus Afros, pars Scythiam et rapidum cretae ueniemus Oaxen et penitus toto diuisos orbe Britannos. Di costoro i Romani conoscono meglio quelli stanziati nelle regioni meridionali,

${ }^{1}$ Su tale periodo e le relative problematiche, si vedano i seguenti lavori: Alcock, 1971; Morris, 1973; Dumville, 1977; Arnold, 1984; Jones, 1998; Snyder, 1998; Collins - Gerrard, 2004.

${ }^{2}$ Per un quadro della cristianità britannica fino all'avvento di Agostino, vedi McNeill, 1974

e Herren - Brown, 2002.

${ }^{3}$ Cfr. Santoro, 1991 e Luiselli, 1992: 133 ss.

${ }^{4}$ Historia Romana II 46, 1

${ }^{5}$ Virgilio, Bucolica, I 67. 
secondo Tacito particolarmente affini ai Galli (proximi Gallis et similes sunt) ${ }^{6}$. E nello stesso luogo, lo storico romano, ricalcando la varietà dei popoli che vivono in Britannia, scrive che i Caledoni sembrano di origine germanica per il rosso dei capelli e la robustezza delle membra, laddove i Siluri del Galles meridionale sono scuri, simili agli Iberi.

Più ci si avvicina al Vallo di Adriano e più confuse divengono le conoscenze delle terre ulteriori. Di là sono i barbari ${ }^{7}$, e a partire soprattutto dalla fine del IV secolo molti di quei barbari si riversano sulla Britannia romana, attaccando le guarnigioni di stanza alle frontiere settentrionali dell' isola: sono i Pitti e gli Scoti, che il britanno Gildas (fine secolo V - ca. 570) così definisce: tetri [...] greges, moribus ex parte dissidentes, sed una eademque sanguinis fundendi aviditate concordes. Significativamente, lo stesso Gildas li rammenta come gentes transmarinae vehementer saevae, per rilevare che dalla Britannia i due popoli sono separati dall' istmo formato dai fiumi Clyde e Forth' e, insieme ai Sassoni, sono proprio i suddetti due popoli i massimi responsabili della distruzione della Romanitas in Britannia ${ }^{10}$. La loro alleanza è peraltro testimoniata anche da reperti archeologici che confermano scambi commerciali tra Irlanda e Scozia già nel sec. IV in territori abitati da tribù appartenenti a queste genti $^{11}$. Innumerevoli i massacri perpetuati, con i superstiti deportati nella parte occidentale dell'isola (Cornovaglia e Galles), non di rado insieme a quei cristiani che nelle medesime regioni fonderanno scuole e monasteri alla metà del $\mathrm{V}$ secolo ${ }^{12}$. Le loro scorrerie "ufficiali", accompagnate da quelle degli Attacotti irlandesi e, da est, dei Sassoni medesimi, hanno luogo nel $360^{13}$, e ancora nel $365^{14}$.

Gli Attacotti (celt. *Ate-scotti, con un prefisso peggiorativo combinatosi all'etnonimo Scotti, atto evidentemente a rimarcare un gruppo tribale particolarmente non gradito per i suoi costumi, dunque tenuto separato ${ }^{15}$ ) furono definiti bellicosa hominum natio dallo stesso Ammiano ${ }^{16} \mathrm{e}$ avevano le loro sedi in Irlanda ${ }^{17}$.

\section{${ }^{6}$ Agricola 11, 2}

${ }^{7}$ Sulle conoscenze e la concezione romane dei barbari, vedi ancora Luiselli, 1992: 383-406; cfr. pure Borca, 2000: 117-119; 123-124.

${ }^{8}$ De excidio et conquestu Britanniae 19, ed. T. Mommsen, in Chronica minora saec. IV-VII, III, MGH, Auct. ant. XIII (Berlin 1898), 35, 11. 10-11.

${ }^{9}$ De excidio et conquestu Britanniae 14 . Quest' ultima notizia sarà poi ripresa da Beda: Historia Ecclesiastica gentis Anglorum I, 12, edd. B. Colgrave - R.A.B. Mynors, Bede's Ecclesiastical History of the English People (Oxford, 1991), 40. Sulla questione, vedi Wright, 1984.

${ }^{10}$ Così, infatti, lascia intendere ancora Gildas (De excidio et conquestu Britanniae 24). Sulla questione, vedi Higham, 1994: 43.

${ }^{11}$ Cfr. Laing - Laing, 1986.

${ }^{12}$ Cfr. Burns, 1991: 72.

${ }^{13}$ Ammiano Marcellino, Res gestae XX 1, 1.

${ }^{14}$ Ammiano Marcellino, Res gestae XXVI 4, 5.

${ }^{15}$ Freeman, 2001: 127.

${ }^{16}$ Res gestae XXVII 8, 5 .

${ }^{17}$ Cfr. Cunliffe, 1997: 262-263; Freeman, 2001: 95-96, 98-100; Rance, 2001. 
Costoro erano noti anche a Girolamo, che in uno scritto del $393 \mathrm{ca}$. lascerebbe intendere di averne visti alcuni in Gallia, rilasciandone un giudizio non certo lodevole: Quid loquar de caeteris nationibus, cum ipse adolescentulus in Gallia Atticotos, gentem Britannicam, humanis vesci carnibus'; Scottorum et Aticottorum ritu ac de Republica Platonis promiscuas uxores, communes liberos habeant ${ }^{19}$. In ultimo, la Notitia Dignitatum (in partibus Orientis IX; in partibus Occidentis V, VII), documento compilato nel 400 ca., registra presenze di ausiliari attacotti nell'esercito romano (Honoriani Atecotti seniores) in terre galliche, illiriche e italiche alla fine del IV secolo ${ }^{20}$.

Due anni dopo le suddette scorrerie, nel 367, si realizza il triplice attacco sferrato simultaneamente ancora dalle medesime genti, in buona parte frenate dal comes Flavio Teodosio, padre dell'imperatore ${ }^{21}$. E altre incursioni si susseguono tra il 368 e il $369^{22}$, mentre più avanti, nel 382 , secondo un'omonima cronaca gallese del 452, il vir probus Magno Massimo vince i Pitti e gli Scoti, così da essere proclamato imperatore l'anno seguente proprio dall'esercito dislocato in Britannia ${ }^{23}$. Tra il 396 e il 399, sulla costa occidentale dell' isola, è Stilicone a respingere una di queste massicce incursioni ${ }^{24}$; nel 407 il municeps Graziano è eletto tiranno, ma, presto ucciso, è sostituito da Costantino, il quale, dopo avere usurpato il titolo di imperatore e avere condotto le truppe dalla Britannia in Gallia, finisce per essere assassinato su mandato di Onorio ${ }^{25}$. Nel corso del sec. V i Britanni sono così costretti a chiedere sovente aiuto ai Romani ${ }^{26}$, anche perché i Pitti stringeranno presto alleanze con Sassoni e Angli ${ }^{27}$.

L'anno preciso dell'evacuazione romana non può essere determinato con esattezza, ma tra il 407 e il 411 la Britannia è svincolata da Roma, anche sulla scia del rescritto di Onorio del 410, con il quale le poleis britanniche venivano autorizzate ad amministrarsi da sole ${ }^{28}$. Alla metà del $\mathrm{V}$ secolo, vale a dire negli anni in cui fiorisce san Patrizio, la Britannia è dunque un paese indipendente, governato

${ }^{18}$ Girolamo, Adversus Jovinianum II, 7, PL 23, 296 A.

${ }^{19}$ Girolamo, Epistula 69 (Ad Oceanum), 3 (ed. J. Labourt, Paris, 1953, 196, 5-6 = CSEL 54, 684, 11. 6-14).

${ }^{20}$ Notitia dignitatum: accedunt Notitia urbis Constantinopolitanae et Laterculi provinciarum, ed. O. Seeck, Berlin 1876, pp. 29, 124, 133,

${ }^{21} \mathrm{Si}$ tratta della cosiddetta barbarica conspiratio ricordata da Ammiano Marcellino (Res gestae XXVII, 8, 1-5, ed. M.-A. Marié, Paris 1984, III, 126-127). Cfr. Morris, 1973: 15 ss.; Rankin, 1987: 123 ss.

${ }_{22}$ Ammiano Marcellino, Res gestae XXVII, 8.

${ }^{23}$ Chronica Gallica, ed. T. Mommsen, Chronica Minora saec. IV. V. VI. VII., I, MGH Auct. Antiq. IX, 1892, 629-666, qui 646. La stessa notizia leggiamo in Gildas (13) e Beda I 9, edd. Colgrave-Mynors, cit., p. 36. A proposito si vedano Dillon - Chadwick, 1968: 58; e Rankin, 1987: 224-227.

${ }^{24}$ Claudiano, De consulatu Stilichonis II 247-255. La stessa notizia è ricavabile anche dalla lettura di Gildas, 14-20, 33-36. Cfr. Byrne, 1973: 76; Thomas, 1981: 286, 317.

${ }_{25}$ Beda I 11, edd. Colgrave-Mynors, cit., pp. 38-40.

${ }^{26}$ Ibid. I 12-13, 40-4 (in parte Beda si rifa a Gildas 14-20).

${ }^{27}$ Ibid. I 15, 48-52.

${ }^{28}$ Zosimo, Historia Nova v, 27. 
da principi celtici altrettanto indipendenti ${ }^{29}$, sicché il ritiro delle legioni riporta la nobiltà indigena a una restaurazione pure agevolata dal persistere di un ordine sociale celtico mai sopitosi negli anni della romanizzazione ${ }^{30}$.

La prima menzione storica dei Pitti è in un panegirico del 297, dove, insieme agli Irlandesi (Hiberni), sono descritti come acerrimi nemici dei Britanni ${ }^{31}$. In effetti, tale etnonimo indica genericamente tutti gli abitanti delle terre a nord del già ricordato fiume Clyde $^{32}$, rimasti pertanto al di là della frontiera romana, in extrema parte insula ${ }^{33}$. Considerati da non pochi studiosi i discendenti di quelle tribù di Celti misti a indigeni che, detti in genere Caledonii (o Caledones) $)^{34}$, nel corso dell'età di Hallstatt (prima Età del Ferro, 700-500 a.C.) risiedevano nella Scozia centrosettentrionale ${ }^{35}$, di fatto i Pitti occupavano quasi per intero l'attuale Caledonia ${ }^{36}$. È opportuno ricordare che ovunque i Celti fossero stanziati è possibile costatare la permanenza e l'incorporamento degli elementi autoctoni nei nuovi organismi sociali e politici, ai primi sovrappostisi, benché in una forma sociale che provocherà quell'assenza di unità che risulterà letale per la sopravvivenza di questo popolo; così, infatti, scrive Tacito relativamente ai Britanni:

Olim regibus parebant, nunc per principes factionibus et studiis trahuntur. Nec aliud adversus validissimas gentis pro nobis utilius quam quod in commune non consulunt. Rarus duabus tribusve civitatibus ad propulsandum commune periculum conventus: ita singuli pugnant, universi vincuntur ${ }^{37}$.

Stando al racconto di Beda ${ }^{38}$, dopo aver guadagnato in origine la Britannia settentrionale, i Pitti giunsero in Irlanda e chiesero ai gaelici Scoti dei territori dove stabilirsi. Su consiglio di questi ultimi, quelli si insediarono nel nord della Britannia, dal momento che la parte meridionale era già abitata da genti indigene o protoceltiche. Inoltre, non avendo mogli le chiesero agli stessi Scoti, ottenendole a condizione di scegliere il proprio re dalla linea regia femminile ${ }^{39}$. Lo steso Beda aggiunge

${ }^{29}$ Procopio, De bello vandalico I, 2 (= De bellis III 2, edd. J. Haury - G. Wirth, I, Leipzig 1962). Cfr. Morris, 1973: 43-44.

${ }^{30}$ Vedi Alcock, 1971: 88-113; Smyth, 1984: 1-35; Iannello, 2013 b.

${ }^{31}$ Incerti Panegyricus Constantio Caesari Dictvs XI, 4, ed. E. Galletier, Panégyriques Latins tome II (I-V) (Paris 1949), 91. Si veda anche Incerti Panegyricus Constantini Augusti VII, 2, ed. E. Galletier, Panégyriques Latins tome II (VI-X) (Paris 1952), 59.

${ }^{32}$ Foster, 2004: 11.

${ }^{33}$ Gildas 21.

${ }^{34}$ Powell, 1959: 173-176; MacKie - MacKie, 1984. Vedi pure Dillon - Chadwick, 1968: 54-56, 111 ss.

${ }^{35}$ Foster, 2004: 11-13.

${ }^{36}$ Hubert, 1932: 247-249.

${ }^{37}$ Agricola 12, 2-4.

${ }^{38}$ Historia ecclesiastica gentis Anglorum I 1, edd. Colgrave-Mynors, cit., p. 18.

${ }^{39}$ Sulla sovranità matrilineare dei Pitti, vedi Miller, 1982. 
che i Pitti sarebbero discesi dal nord, probabilmente dalle regioni scandinave centromeridionali ${ }^{40}$, ma il problema relativo alla loro genesi rimane ancora insoluto, malgrado le numerose e diverse ipotesi avanzate da archeologi, filologi ed etnologi ${ }^{41}$. Isidoro di Siviglia spiega l'origine del loro nome dal costume di dipingersi il corpo ${ }^{42}$ ("il popolo dei disegni" o "popolo tatuato": gens Pictorum, nomen a corpore, quod minutis opifex acus punctis et expressus native graminis sucus inludit) e, già prima, Cesare testimoniava che i Britanni dell'interno dell'isola si pitturavano il corpo prima di andare in guerra (Omnes vero se Britanni vitro inficiunt, quod caeruleum efficit colorem, atque hoc horribiliores sunt in pugna adspectu $)^{43}$. Si può dunque supporre che i Celti della Britannia meridionale avessero appreso l'usanza di dipingersi o tatuarsi da popolazioni a loro preesistenti. Ancora in Beda ${ }^{44}$, la loro lingua è annoverata tra le quattro parlate in Britannia, insieme a quelle di Britanni, Scoti e Angli, mentre nell' Historia Brittonum, il corpus pseudo-storico compilato dal monaco gallese Nennio tra 829 e 830, sulla base di un testo in origine risalente con molta probabilità alla fine del secolo VII ${ }^{45}$, leggiamo che la Britannia insula è abitata da quattro popolazioni: Scoti, Pitti, Sassoni, Britanni ${ }^{46}$.

La diffusione dei Pitti è pure testimoniata dal nome delle Isole Britanniche. I Britanni sono detti infatti Prydain dai Gallesi, da un originario * Pritani, sì che Ynys Prydain divenne il nome delle Isole Britanniche nel loro insieme ${ }^{47}$, perfetto

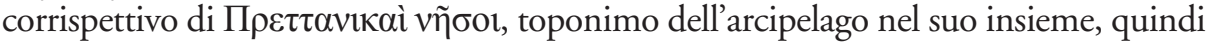
comprendente anche 'Í́pvm (l'Irlanda) e per la prima volta trasmessoci nella seconda metà del IV secolo a.C. dall'oscuro navigatore e geografo Pitea di Marsiglia nel Пєpì

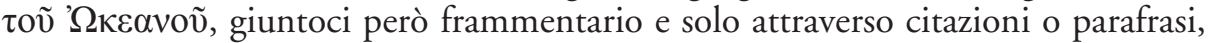
perlopiù indirette, di autori più tardi ${ }^{48}$. Da qui, Dionigi Periegeta (I-II sec.) chiamerà i suoi abitanti con la variante successiva di B $\rho \varepsilon \tau \alpha v o i ̀ ~ o ~ B \rho \varepsilon \tau \alpha v i ́ \delta \varepsilon \varsigma^{49}$.

${ }^{40}$ Historia ecclesiastica gentis Anglorum I, 1, edd. Colgrave-Mynors, cit., p. 16, dove l'autore, quasi certamente per errore, scrive Scythia in luogo di Scandia.

${ }^{41}$ Si vedano O'Rahilly, 1946: 341-384; Wainwright, 1958; Sutherland, 1994); Foster, 2004.

${ }^{42}$ Etymologiae XIX 23, 7.

${ }_{43}$ De bello gallico V 14, 2. Vedi pure Marziale, Epigrammata XI, 53 e XIV, 99, e Isidoro XIX, 23, 7 (stigmata Brittonum).

${ }^{44}$ Historia ecclesiastica gentis Anglorum III, 6, edd. Colgrave-Mynors, cit., p. 230; vedi anche I 1, 16, dove viene aggiunto il latino.

${ }^{45}$ Cfr. Dumville, 1972-1974; Id., 1975-1976:; 78-95; Luiselli, 2003: 281.

${ }^{46}$ Historia Brittonum 7, ed. Mommsen. 147, 11. 9-10.

${ }^{47}$ Loth, 1920-1921: 280, n. 4; Hubert, 1932: 248; O’Rahilly, 1946: 451; Freeman, 2001: passim.

${ }^{48}$ Per le specifiche informazioni piteane sopra riportate concernenti le Isole Britanniche, vedi Timeo, fr. 164 Jacoby, 1. 404, e Diodoro, Bibliotheca V, prol., 1; v 21, 2.5; v 22, 1-3; v 38, 5.

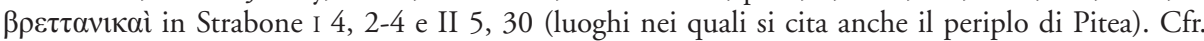
Magnani, 2002: 131-133. Vedi anche Gougaud, 1907; Loth, 1920-1921: 280 ss.; O'Rahilly, 1946: 341 ss.; 445 ss.; Sergi, 1987: passim; Luiselli, 1992: 99 ss.; Freeman, 2001: passim. Per la menzione dell'Irlanda nei frammenti ascrivibili alla tradizione piteana, vedi Fragmenta, 6a, 19.44.53; 6b, 33; 6c, 21.51, ed. H. J. Mette, Berlin 1952.

${ }^{49}$ Orbis descriptio 284; 566; cfr. 569. 
Molto più avanti, tra IV e V sec., così riassumeva talune nodali e diffuse informazioni piteane sulle Isole Britanniche il geografo Marciano di Eraclea, in una specifica sezione del suo Periplus maris exteri (II 41), la più vasta opera geografica dell'antichità dopo quella di Claudio Tolemeo (II sec.):

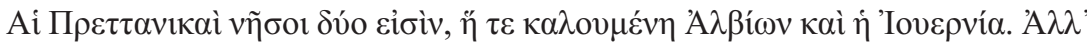

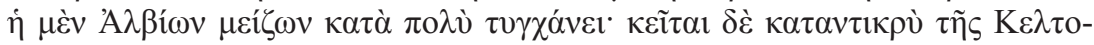

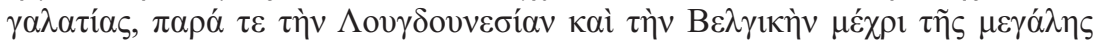

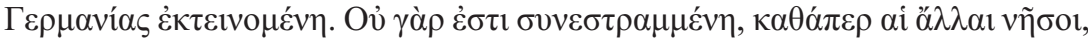

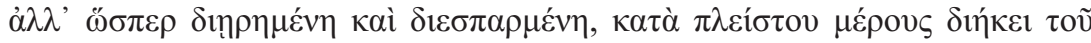

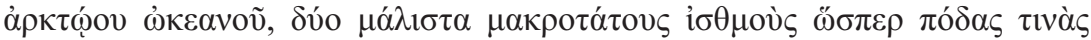

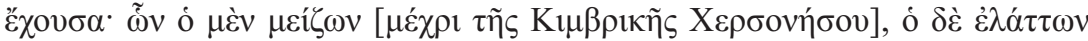

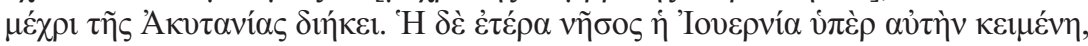

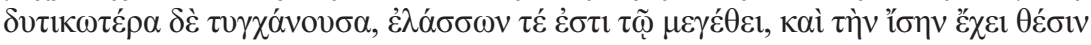

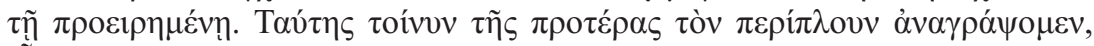

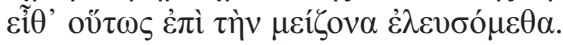

Le parole e le forme or ora scorse si legano evidentemente al gallese pryd e all'antico-irlandese cruth ("figura/forma") 50 , da cui Cruthen-tuath, o Cruithentuath, loro nome più specifico ${ }^{51}$. In irlandese, allora, il termine invalso per designarli è Cruithnig, o Cruithin, usato per indicare distintamente una parte degli abitanti della Britannia e dell'Irlanda ${ }^{52}$. Nella Vita Columbae di Adomnano, abate di Iona dal 679 al 704 (anno della sua morte), i Pitti della Caledonia vengono infatti chiamati Cruthini populi, letteralmente "popolo di Cruthen", l'eponimo dei Cruithni ${ }^{53}$. Tutto ciò si è verificato perché, com'è noto, il suono velare q- del gaelico, esito della labiovelare sorda indoeuropea ${ }^{*} k w$-, progredì nel labiale $p$ - del ramo brittonico ${ }^{54}$. È così possibile concludere che il nome Picti derivasse in origine da una radice analoga a quella dell'antico-irlandese cicht ("incisore") ${ }^{55}$.

Riguardo all'etnonimo Scotti, sebbene la sua etimologia permanga parzialmente oscura, esso, di fatto, può essere associato al verbo antico-irlandese scothaim, che indica un movimento veloce, istantaneo e freddo, "a sorpresa", allora. Esso può così assumere il significato meno letterale di "invadere" e/o "saccheggiare" e, nel

${ }^{50}$ Hubert, 1932: 247-249. Vedi il latino cerussa, con il quale si indica una sostanza colorante bianca detta "biacca di piombo" o, più semplicemente, "cipria": Plauto, Mostellaria 258 e 264; Ovidio, Medicamena faciei femineae 73; Marziale, Epigrammata, I 72, 6; II 2, 41; VII 25, 2; X 22, 2; Plinio XXVIII passim.

${ }^{51}$ Loth, 1920-1921: 280, n. 2; Rankin, 1987: 252.

${ }^{52}$ O’Rahilly, 1946: 431-433; 444-52.

${ }^{53}$ Ed. A. O. Anderson - M. O. Anderson, Adomnan's Life of Columba, Oxford 1991, 50a, 88; cfr. 18a, 30).

${ }^{54}$ Vedi Sims-Williams, 1993: 380.

${ }^{55}$ Cfr. Hubert, 1932: 249. 
moderno inglese, ritrovare analogie nell'aggettivo scathing ("feroce"). La stessa radice indoeuropea *skath si legge nel greco skedannumi ("disperdere", "spargere"), nel latino seco, e nei sostantivi antico-irlandesi scáth e scál, che non casualmente indicano l'ombra (ingl. shadow), e scían, "coltello" e "lama"s6. Nel Lebor Gabála Érenn ("Libro delle Invasioni d'Irlanda") $)^{57}$, Scota è la moglie di Míl, i cui quattro figli, i cosiddetti Milesi, sarebbero i progenitori degli Irlandesi attuali, come Gaedel Glas, figlio della stessa, lo sarebbe dei Gaeli in genere ${ }^{58}$. E ancora, Scáthach (irl. "ombrosa") è il nome di un'eroina del ciclo mitologico dell'Ulster, una guerriera soprannaturale che prepara i giovani alla guerra, compreso il celebre Cú Chulainn ${ }^{59}$.

Con Scotti, allora, possono genericamente intendersi dei corsari, dei saccheggiatori, insomma degli organizzatori di vere e proprie spedizioni militari: è infatti certo che essi praticassero sovente il brigantaggio ${ }^{60} \mathrm{e}$, peraltro, tali scorrerie, così come la stessa presenza di prigionieri britannici nell' isola, fungevano da canale di penetrazione per la cultura latina ${ }^{61}$. L'etnonimo in questione lo si ritrova per di più anche nell'onomastica gallica, dove indica esattamente territori abitati da pirati ${ }^{62}$. E le abitudini scarsamente sobrie degli stessi sono sottolineate anche da san Girolamo, quando li descrive come pagani viventi in promiscuità ${ }^{63}$. L'appellativo Scotti, pertanto, il quale, e forse troppo spesso, è superficialmente utilizzato per definire delle bande di avventurieri $^{64}$, in origine indicava decisamente dei predoni, dei razziatori. E gli Scoti dell'Argyll, al confine con lo Strathclyde ${ }^{65}$, poco più a nord della penisola di Cowal,

${ }^{56}$ Vendryes, 1974: 42 ss.; de Bernardo Stempel, 1999: 254; cfr. pure MacNeill, 1920: 145-148. Si vedano anche i sostantivi latini scena e sacena in Sesto Pompeo Festo, De verborum significatione, ed. W. M. Lindsay, Leipzig, 1913, 318, 1l. 50-51, e 422, 1. 32, dove indicano la scure per i sacrifici (dolabra pontificalis: cfr. J. Yates, s.v. Dolabra, in Smith, 1875: 420).

${ }^{57}$ Opera composta nel XII secolo, ma che ha origine da compilazioni di monaci effettuate già a partire dal VI secolo, al fine di redigere una storia mitica delle origine del popolo gaelico d'Irlanda. Si veda l'edizione di R.A.S. Macalister, Lebor Gabála Érenn. The Book of the Taking of Ireland, 5 voll., Dublin, 1938-1956.

${ }^{58} \mathrm{Si}$ veda in O'Rahilly (1946) il capitolo The Goidelic Invasion: 193-208. Cfr. anche Rees Rees, 1961: 26-41; 95-117; Le Roux, 1968: 399 ss.; Markale, 2001: 153 ss.; Rankin, 1987: 13-14; 27-28; Luiselli, 2003: 95-96. Ancora sulle invasioni dell'Irlanda, si veda il resoconto leggendario in Historia Brittonum 13, ed. Mommsen, 154-156.

${ }^{59}$ Si vedano O’Rahilly, 1946: 61; Rees - Rees, 1961: 254 ss.; Olmsted, 1982.

${ }^{60}$ Cfr. Haverfield, 1913: 8; Mac Neill, 1920: 145-148; Malaspina (ed.), 1985: 129, n. 324; Iannello, 2013b. Chiaramente a costoro si riferisce Gildas quando definisce gli Hiberni in genere impudentes grassatores $(21,1)$

${ }^{61}$ Malaspina, 1985: 3.

${ }^{62}$ Cfr. Haverfield, 1913: 8.

${ }^{63}$ Adversus Jovinianum II, 7, PL 23, 296 A: Scotorum natio uxores proprias non habet, Epistula LXIX, 3, Ad Oceanum, ed. J. Labourt (Paris, 1953) III, 196, 11. 5-7: promiscuas uxores, communes liberos habeant, anche CSEL 54, 684, 14.

${ }^{64}$ Cfr. Kenney, 1929: 79; Powell, 1959: 177; Anderson, 1982: 106-132, passim.

${ }^{65}$ Cfr. Beda, Historia ecclesiastica gentis Anglorum I 1, edd. Colgrave - Mynors, cit., p. 20. Si veda Thomas, 1981: 342 . 
erano effettivamente dediti ad azioni piratesche ${ }^{66}$ : latrunculi, li chiama Patrizio in Epistola ad milites Corotici 12, 3, nonché hostes (Confessio 46, 1167), cioè estranei alla Romania cristiana ${ }^{68}$. Inoltre, possedendo questi delle basi dislocate lungo le coste del Galloway, si può bene ipotizzare che pirati scoti residenti nell'Irlanda settentrionale, misti a Pitti stanziati in Irlanda e a rifugiati di origine britannica ${ }^{69}$, avessero attaccato la uillula di Calpornio, padre di Patrizio, nei pressi di Bannauem Taburniae (Bannaventa Berniae), non molto distante dalla costa britannica d'occidente ${ }^{70}$, e poi rapito il santo, allora sedicenne (Confessio 1, 4-8). Anche la stessa cattura di Patrizio e dei suoi comites al tempo della missione itinerante (Confessio 52) potrebbe essere stata attuata da gruppi di latrones. Gli Scoti indigeni irlandesi convissero certo a lungo con i Pitti dell'Irlanda settentrionale, tra i quali si contavano dei nati da rifugiati britanni. Non di rado, i due popoli giungevano a combinarsi attraverso il matrimonio $^{71}$ e insieme operavano incursioni nella Britanni romana, a sud del Vallo di Adriano.

\section{HIBERNIA}

Le prime notizie certe sull'Irlanda ci provengono dai rapporti di alcune navigazioni databili a partire dal VI sec. a.C. Si tratta dei peripli compiuti dal massaliota Eutimene e dal cartaginese Imilcone (530 a.C.), nonché del già ricordato viaggio nell'estremo nord di Pitea (IV sec. a.C.). Dei primi due esistono solo pochi frammenti tramandati da Rufo Festo Avieno (IV sec.) nel poema Ora maritima. Le informazioni dei due navigatori si basano sui racconti dei mercanti di Tartesso (cittadina iberica presso le foci del Guadalquivir, che danno nel golfo di Cadice), i quali, attraverso la rotta dello stagno $^{72}$, già da parecchi anni effettuavano scambi commerciali a nord con gli abitanti delle isole Estrimnidi, collegabili alle sfuggenti Cassiteridi ${ }^{73}$ e identificabili con l'arcipelago posto a sud-ovest della Cornovaglia, quello delle Isole Scilly, o comunque con isole dell'area della costa atlantica dell'attuale Bretagna, precisamente nel golfo di Brest ${ }^{74}$ :

${ }^{66}$ Cfr. Kenney, 1929: 149; Mac Neill, 1920: 145-148.

${ }^{67}$ Ed. R. P. C. Hanson (Saint Patrick, Confession et Lettre à Coroticus. Introduction, texte critique, traduction et notes, Sources Chrétiennes 249, Paris 1978).

${ }^{68}$ Malaspina (ed.), 1985: 75, n. 13, 125, n. 305.

${ }^{69}$ Cfr. Malaspina, 1984: 86.

${ }^{70}$ Cfr. Thomas, 1986: 118.

${ }^{71}$ Vedi Miller, 1982.

${ }^{72}$ Strabone, Geographia, II, 5, 15; III, 5, 11.

${ }^{73}$ Vedi Dion, 1952; Magnani 2002: 115-127.

${ }^{74}$ Cfr. Luiselli, 1992: 66-73; Antonelli, 1998: 156-157; Magnani, 2002: 119. Sulle relazioni in genere tra la penisola iberica e i "mari celtici" nel mondo antico, vedi Maya González, 1999. Ad oggi, il lavoro più completo e di maggior riferimento per la raccolta e lo studio delle fonti greche e latine 


\section{[...]}

Et prominentes hic iugi surgit caput,

Oestrymnin istud dixit aevum antiquius,

Molesque celsa saxei fastigii

Tota in tepentem maxime vergit notum.

Sub huius autem prominentis vertice,

Sinus dehiscit incolis Oestrymnicus,

in quo insulae sese exerunt Oestrymnides

laxe iacentes et metallo divites

stanni atque plumbi multa uis hic gentis est,

superbus animus, efficax solertia,

negotiandi cura iugis omnibus,

netisque cumbis turbidum late fretum

et beluosi gurgitem Oceani secant.

Non hi carinas quippe pinu texere

et acere norunt, non abiete, ut usus est,

curvant faselo(s), sed rei ad miraculum

navigia iunctis semper aptant pellibus

corioque vastum saepe percurrunt salum.

[...]

$[\ldots]$

$[\ldots]$

[...]

Tartes(s)iisque in terminos Oestrumnidum

negotiandi mos erat. $[\ldots]^{75}$

Ed è incastonato nei quattro versi che precedono gli ultimi due di questa sezione "nord-atlantica" che si può leggere quello che può essere considerato il più antico riferimento storico all'Irlanda, citata a proposito di traffici tra gli stessi Estrimnii e gli Hierni, stanziati in un' insula detta sacra e prospiciente Albione (la Britannia) ${ }^{76}$ :

dell'antica Irlanda è quello, già citato, di Freeman, 2001. Analoghe notizie su rapporti commerciali sono tramandate da Tolemeo in merito agli scambi intrattenuti dai mercanti di La Coruña con i Luceni e i Velabri, popolazioni stanziate presso l'estuario dello Shannon, il maggiore fiume irlandese (Geographica, II, 2, 34. Cfr. Powell, 1959: 22-25; Malaspina, 1984: 30-38; Luiselli, 1992: 66-73; 99-130, passim).

${ }^{75}$ Avieno, Ora maritima, 90-107; 113-114, ed. A. Schulten, Avieni Ora maritima (periplus Massiliensis saec. VI a.C.) adiunctis ceteris testimoniis anno 500 a.C. antiquioribus, Barcinone-Berolini 1922, pp. 57-58.

${ }^{76}$ Albion in Plinio, Naturalis Historia IV, 102, 4 E 111, 6; Labeon in Apuleio, De Mundo 301,

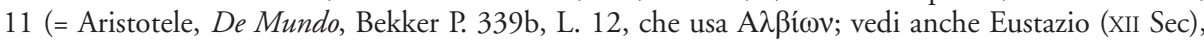
Commentarium In Dionysii Periegetae 566, 3; Marciano, Periplus Maris Exteri I, 8, 4; II, A, 20; 1, 14; 41, 2; 44t, 1; 44, 1-2; 45, 1 E 14; Stefano di Bisanzio, Ethnica, 69, 16-17; Strabone VII, 5, 4). Attestata

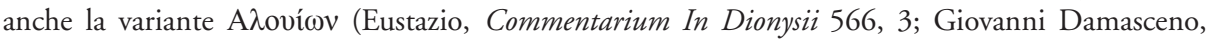
Expositio Fidei 24b, 1. 2; Filostorgio (II-V Sec), Historia Ecclesiastica I, 5, 5; Tolemeo, Geographica II, C, 2; 3, T; 3, 14; VII, 5, 11; VIII, 3, 6; 29, 1, ed. K. Müller, Paris 1883). 
Ast hinc duobus in sacram sic insulam

dixere prisci solibus cursus rati est

haec inter undas multam caespitem iacet

eamque late gens Hiernorum colit

propinqua rursus insula Albionum patet ${ }^{77}$.

Fu dunque lungo la rotta dello stagno, nel sec. VI a.C., che si sarebbe diffusa

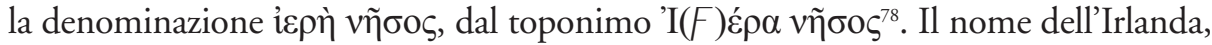
infatti, assodati i naturali esiti assonanzati nei toponimi adottati dalle genti di lingua greca, avrebbe origine dal proto-celtico ${ }^{*}(f)$ iwerjon, nella radice indoeuropea identico

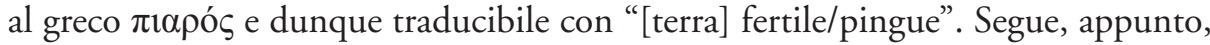
il proto-irlandese *Euerio, che dà la forma antico-irlandese Eriu (gallese Iwerddon); l'isola era anche detta Erinn, al dativo (genitivo Erenn), ed oggi Éire $e^{79}$.

Le forme greche 'Ićpv $\eta^{80}$ e 'Iovés $\rho \eta^{81}$, alle quali rimanda peraltro l'etnonimo

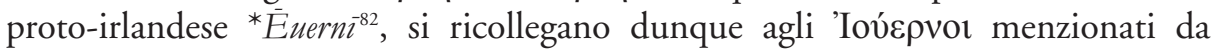
Claudio Tolemeo ${ }^{83}$, che ne ricava il toponimo 'Iovepví $\alpha$ per indicare l'intera isola ${ }^{84}$. Seguono, così, i corrispondenti latini Iuuerna ${ }^{85}$ e Iuerne ${ }^{86}$, Iuuernae/Hiuerne/Iuerne ${ }^{87}$ e il più comune, invero forma divenuta universale, Hibernia, poi grecizzato in 'I $\beta \varepsilon \rho v i \alpha^{88}$, nome dell'isola che certamente risente anche dell'influsso di hibernus ${ }^{89}$,

${ }_{77}$ Avieno, Ora maritima 108-112, ed. cit., p. 58. Su queste notizie si veda il commento di Antonelli, 1998: 35 ss., 48, 80, 111, 118, 156-157.

${ }^{78}$ Vedi Rankin, 1987: 6-8; Rankin, 1995; Cunliffe, 1997: 149 ss.

${ }^{79}$ Vedi Holder, 1896-1907: II, s.v. Iueriu, col. 99; Hogan, 1910: 400; Hubert, 1932: 243; Pokorny, 1959: 324; Koch, 1990; de Bernardo Stempel, 1999: 114.

${ }^{80}$ Aristotele, De mundo, ed. Bekker, p. 393b, 1. 13; Geographica adespota fragmenta, fr. 1, 1. 7, ed. K. Müller, Geographi Graeci minores, vol. 2. Paris 1861, repr. Hildesheim 1965, pp. 509-511; Elio Erodiano e pseudo-Erodiano, De prosodia catholica 3, 1, p. 327, 1. 26; Ipparco, Fragmenta geographica, fr. 61 1.; Orphica Argonautica, ed. G. Dottin, Paris 1930, 1. 1181; Tolemeo, Geographica II, 2, 3, 1. 10; Pitea, Fragmenta 6a, 19, 44, 53; 6b, 33; 6c, 21 e 51; Stefano di Bisanzio, Ethnica, p. 328, 1. 14; Giovanni Stobeo, Anthologium I 40, 1, 132, ed. C. Wachsmuth - O. Hense, Berlin 1884.

${ }^{81}$ Stefano di Bisanzio, Ethnica, ed. A. Meineke, Graz 1958, p. 335, 1. 1.

${ }^{82}$ Cfr. Strabone, Geographica I 4, passim.

${ }^{83}$ Geographica II 2, 6.

${ }^{84}$ Tolemeo, Geographica I, 11, 7, 1-14; II, C, T, 4 - II C, 1-11; II, 2, T, 1; II, 2, 3, 1-11; II, 2, 6, 1-3; II, 2, 7, 1-8; II, 2, 9, 1-8; 10, 1-15; 3, T, 1-2; II, 3, 2, 1-3; VII, 5, 11, 1-8; VIII, 3, 4, 1-5; 5 , 1-3; 6, 1-4; 7, 1-3 (ed. K. Müller, Paris 1883).

${ }^{85}$ Pomponio Mela, De Chorographia III 53; Giovenale, Satura II 159.

${ }^{86}$ Claudiano, Panegyricus Dictus Honorio Augusto Quarto Consuli 33, ed J. B. Hall, Leipzig 1985, p. 62; Id., De Consulatu Stilichonis II 251, ed. Hall, cit., p. 214.

${ }^{87}$ Cfr. Claudiano, De IV consulatu Honorii Augusti, vIII, 33.

${ }^{88}$ Eustazio, Commentarium in Dionysii, 566, 3; cfr. Tolemeo, Geographica, VIII 29, 1.

${ }^{89}$ Cfr. O’Rahilly, 1946: 451, n. 3. 
e attestato già da Cesare (De Bello Gallico V 13, $2^{90}$ ). Si ricordi poi la singolare forma Iberi usata da Colombano per designare in genere gli abitanti dell'Irlanda ${ }^{91}$.

Negli scritti di san Patrizio l'isola è denominata Hiberione ${ }^{22}$, forma unica e indeclinabile che, come scrive Elena Malaspina, rappresenterebbe un accusativo sclerotizzato ricalcato sull'accusativo protoirlandese * Ëuĕrĭŏnĕm (o *Iuĕrŏonĕm), caratterizzato dalla perdita della nasale finale e dal santo personalmente recepito in ambiente gaelico ${ }^{93}$, oppure, stando ad Hanson, di un locativo in luogo di un nominativo ${ }^{94}$. In ambito mitico ed epico, Ériu è pure il nome della tradizionale dea-madre d'Irlanda, che, dotata di attributi solari, è connessa alla fertilità e alla funzione regale in qualità di sposa di ogni "re-supremo" dell'isola (ard-rí)

Si ricordi che Tolemeo (libro II), che utilizza fonti certamente molto antiche ${ }^{96}$, inizia la sua descrizione del mondo proprio dall'Irlanda, in quanto considerata la parte più lontana dell'Europa occidentale ${ }^{97}$. Del resto, Patrizio stesso sottolineerà con forza il suo essere usque ad ultimum terrae (Confessio 1, 13; vedi anche Confessio 58 e Epistola ad milites Corotici 9, 8), dunque la natura e la prospettiva periferica, "ulteriore", della sua missione ${ }^{98}$.

Ritornando ancora alla spedizione di Pitea ${ }^{99}$ (325-323 a. C.), bisogna rilevare che egli raggiunse, attraverso la Manica, la Cornovaglia, la Scozia, la costa dell'Irlanda $\mathrm{e}$, forse, alcuni punti della penisola scandinava riconducibili a Thule ${ }^{100}$. Circumnavigata la Britannia, la flotta si avventurò nella perlustrazione interna sul versante irlandese, lungo il canale del nord. È dunque grazie al resoconto di queste navigazioni che l'Irlanda entrò definitivamente nella storia e nella geografia antica, dissolvendo l'alone di mistero e di nebulosità che sempre l'aveva caratterizzata. Anche Strabone, il quale, guardando a Eratostene, pone l'isola a nord della Britannia, ammette che

${ }^{90}$ cfr. Plinio, Naturalis Historia IV 103, 1-5; Tacito, Agricola 24, 1.3; Annales XII 32, 5; Orosio, Historiae I 2.

${ }^{91}$ Epistulae, II, 9 e V, 3, ed.G. S. M. Walker, Sancti Columbani Opera, SLH 2, Dublin 1957, p. $22,1.15$ e p. $38,23$. $1 ; 62,4$.

${ }_{92}^{2}$ Epistola ad milites Corotici 1, 1; 5, 6; 10, 1; 12, 6; Confessio 1, 7; 16, 1; 23, 6; 28, 1; 41,

${ }^{93}$ Malaspina, 1984: 38; vedi anche Bieler, 1951: 89-90.

${ }^{4}$ Saint Patrick: Confession et Lettre à Coroticus, cit., p. 29.

${ }^{95}$ Cfr. Mac Cana, 1955-1956; de Vries, 1991: 161-162; Le Roux, 1968. Fondamentale e poderoso, nonché originale e unico per il tipo di trattazione, il contributo sulla interpretatio christiana delle fonti classiche dell'Irlanda di Ó Corráin, 2010.

${ }^{96}$ Vedi Tierney, 1976, pp. 257-265 e Freeman, 2001: 64-84.

${ }^{97}$ Vedi ancora Freeman, 2001: 68, e, più in genere, 64-84.

${ }^{98} \mathrm{Si}$ vedano in merito gli eccellenti e illuminanti Carey, 1996 e Bisagni, 2011.

${ }_{99}$ Cfr. Strabone, Geographica, II; Plinio, Naturalis historia, IV; Tolemeo, Geographica, II; Avieno, Ora maritima. Vedi Luiselli, 1992: 93-130; Magnani, 2002: passim.

${ }^{100}$ Cfr. Le Roux, 1962; Santarcangeli, 1975: 152 ss.; Levalois, 1985: pp. 6-76; Luiselli, 1992 : 93-130; López Saco, 1994; De Anna, 1998. 
sull'Irlanda (IÉpvๆ), pur già nota come la più grande dell'arcipelago celtico, non si aveva nessuna informazione certa, se non che i suoi abitanti fossero più selvaggi dei Britanni insieme ad altre grottesche, assurde notizie (per es., abitudini antropofaghe e incestuose), benché, invero, lo storico di Amasea sia solerte a evidenziare la non piena affidabilità dei testimoni relativi (Pitea?):



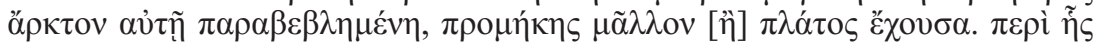

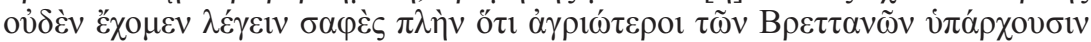

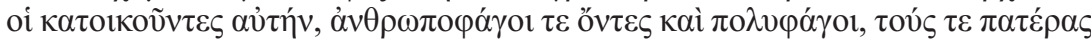

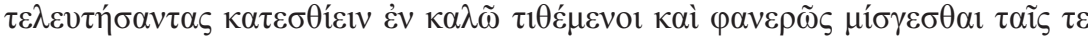

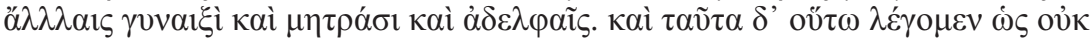

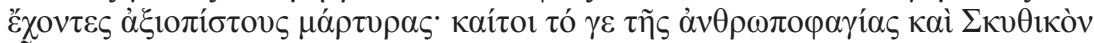

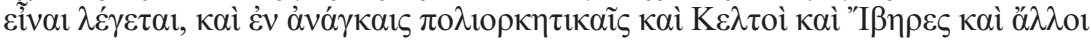

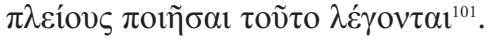

Fonti più tarde sono costituite dalle relazioni delle spedizioni militari di Cesare (Alterum vergit ad Hispaniam atque occidentem solem [scil. Britannia]; qua ex parte est Hibernia dimidio minor, ut existimatur, quam Britannia, sed pari spatio trasmissus, atque ex Gallia est in Britannia) ${ }^{102}$ e, soprattutto, di Agricola:

Quinto expeditionum anno nave prima transgressus ignotas ad id tempus gentis crebris simul ac prosperis proeliis domuit; eamque partem Britanniae quae Hiberniam aspicit copiis instruxit, in spem magis quam ob formidinem; si quidem Hibernia medio inter Britanniam atque Hispaniam sita et Gallico quoque mari opportuna valentissimam imperii partem magnis in vicem usibus miscuerit. Spatium eius, si Britanniae comparetur, angustius, nostri maris insulae superat. Solum caelumque et ingenia cultusque hominu. haud multum a Britannia differunt: melius aditus portusque per commercia et negotiatores cogniti. [...] Saepe ex eo audivi legione una et modicis auxiliis debellari obtinerique Hiberniam posse $[\ldots])^{103}$.

\section{CONCLUSIONI}

Per quanto fin qui detto, una funzione paradigmatica è costituita dalla localizzazione plutarchea dell'oltremondana e iniziatica ${ }^{104}$ Ogigia di Calipso ${ }^{105}$ poco ad

${ }^{101}$ Geographica, IV, 5, 4. Di Strabone vedi altresì Geographica I 4, 3, 5; I 4, 4, 11 ; I 4, 5, 6; II $1,13,13$; II $1,13,15$; II $1,17,13$; II $1,17,16$; II 1,17 , 32; II 1, 17, 34; II 1,17 , 37; II $1,18,14$; II $1,18,25$; II $1,18,27$; II 5,8 , 9; II 5, 8, 39; II 5, 14, 5; II 5, 14, 19; II 5, 14, 38; II 5, 34, 36; IV 5, 4, 2.

${ }^{102}$ Cesare, De bello gallico, v, 13.

${ }^{103}$ Tacito, Agricola 24, 1-5.

${ }^{104}$ Cfr. Le Roux - Guyonvarc'h, 1986: 390.

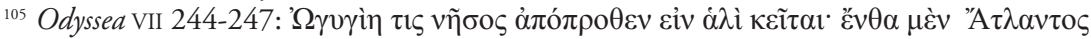

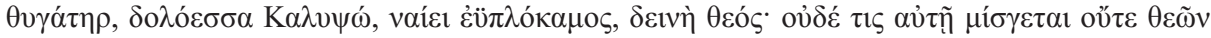

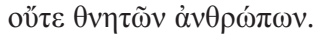


occidente della Britannia, tra isole in linea col tramonto estivo che corrisponderebbero pure alle Fortunatae, ovvero una delle espressioni più celebri di quelle Isole dei Beati generate da una tradizione classica che qui, mitopoieticamente compiendosi, fluisce in quella celtica ${ }^{106}$ :

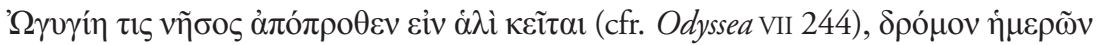

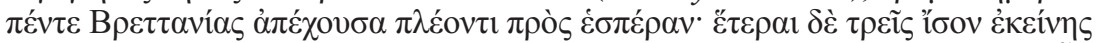

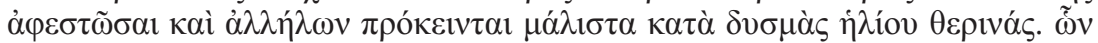

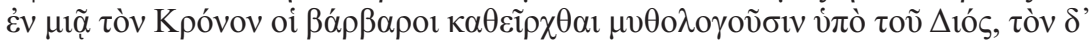

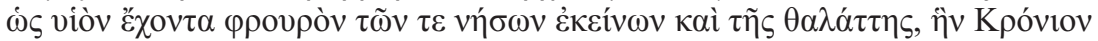

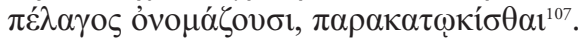

In un celebre passo redatto circa un secolo dopo, dunque alla metà del sec. III, accogliendo e sviluppando quel pregnante concetto di alter orbis con il quale, come sopra già ricordato, Velleio Patercolo acutamente definiva la Britannia, e riprendendo notizie non scevre di mirabilia e paradossi già raccolte da Pomponio Mela due secoli prima $^{108}$, Caio Giulio Solino offre quella testimonianza che in questa sede si rivela decisiva più d'altre perché quanto prepostosi nell'intraprendere il presente contributo possa trovare una sorta di icastico e sostanziale compimento. In tale brano, con toni talvolta ieratici e attraverso le tradizionali connotazioni sacrali già individuate nelle fonti appunto finora considerate, si forniscono descrizioni e considerazioni varie e utili sulle due isole che attraversano le remote asperità della Caledonia e della Cornovaglia per giungere, con una metatemporale e simbolica reductio, a una sacralizzazione dei luoghi citati che significativamente trova la sua manifestazione piena e definitiva nell"' ultima Thule", posta simbolicamente in una direzione quasi "verticale", dunque oltremondana, verso la quale l'Irlanda, ancor più della Britannia, viene a incarnare il ruolo di sacro e ultimo avamposto "orizzontale" ed escatologico d'Occidente:

Finis erat orbis ora Gallici litoris, nisi Brittania insula non qualibet amplitudine nomen paene orbis alterius mereretur: octingenta enim et amplius milia passuum longa detinet, ita ut eam in Calidonicum usque angulum metiamur. In quo recessu Vlixem Calidoniae adpulsum manifestat ara graecis litteris scripta [votum] ${ }^{109}$. Multis

${ }^{106}$ Cfr. Le Roux, 1962; Borca, 2000: 53-69; González Marrero, 2010; Iannello, 2011; Iannello, 2013a: 210-226; Tommasi Moreschini, 2017.

${ }^{107}$ De facie in orbe lunae 941 A 4-11 (ed. M. Pohlenz, Leipzig 1960; si veda anche la più recente edizione con fondamentale commento a cura di P. Donini, Napoli 2011); cfr. Plutarco, De defectu oraculorum 18, e Pindaro, Olympia II, 68-70.

108 Super Britanniam Iuverna est paene par spatio, sed utrimque aequali tractu litorum oblonga, caeli ad maturanda semina iniqui, verum adeo luxuriosa herbis non laetis modo sed etiam dulcibus, ut se exigua parte diei pecora impleant, et nisi pabulo prohibeantur, diutius pasta dissiliant. Cultores eius inconditi sunt et omnium virtutium ignari <magis> quam aliae gentes [aliquatenus tamen gnari], pietatis admodum esperte (Chorographia III 53-54, ed. P. Parroni, Roma 1984, p. 164).

${ }^{109}$ Vedi Zwicker, 1934: 29. Cfr. Tacito, Germania 3. 
insulis nec ignobilis circumdatur. Quarum Hibernia ei proximat magnitudine, inhumana incolarum ritu aspero, alias ita pabulosa, ut pecua, nisi interdum a pastibus arceantur, ad periculum agat satias. Illic nullus anguis, avis rara, gens inhospita et bellicosa. Sanguine interemptorum hausto prius victores vultus suos oblinunt. Fas ac nefas eodem loco ducunt. Apes nusquam: advectum inde pulverem seu lapillos si quis sparserit inter alvearia, examina favos deserent. Sed mare quod inter hanc et Brittaniam interluit undosum inquietumque toto in anno nonnisi pauculis diebus est navigabile idque in centum viginti milia passuum latitudinis diffundi qui fidem ad verum ratiocinati sunt aestimarunt. Siluram quoque insulam ${ }^{110}$ ab ora quam gens Brittana Dumnonii tenent turbidum fretum distinguit. Cuius homines etiamnunc custodiunt morem vetustum: nummum refutant: dant res et accipiunt: mutationibus necessaria potius quam pretiis parant: deos percolunt: scientiam futurorum pariter viri ac feminae ostentant. At Tanatus insula adspiratur freto Gallico, a Brittaniae continente aestuario tenui separata, felix frumentariis campis et gleba uberi, nec tantum sibi verum et aliis salubris locis: nam cum ipsa nullo serpatur angue, asportata inde terra quoquo gentium invecta sit angues necat. Multae et aliae circa Brittaniam insulae, e quibus Thyle ultima, in qua aestivo solstitio sole de cancri sidere faciente transitum nox nulla: brumali solstitio perinde nullus dies. Vltra Thylen accipimus pigrum et concretum mare. Circuitus Brittaniae quadragies octies septuaginta quinque milia sunt. In quo spatio magna et multa flumina, fontes calidi opiparo exculti apparatu ad usus mortalium: quibus fontibus praesul est Minervae numen, in cuius aede perpetui ignes numquam canescunt in favillas, sed ubi ignis tabuit vertit in globos saxeos. Praeterea, ut taceam metallorum largam variamque copiam quibus Brittaniae solum undique generum pollet venis locupletibus, gagates hic plurimus optimusque est lapis: si decorem requiras, nigrogemmeus: si naturam, aqua ardet, oleo restinguitur: si potestatem, attritu calefactus adplicita detinet atque sucinum. Regionem partim tenent barbari, quibus per artifices plagarum figuras iam inde a pueris variae animalium effigies incorporantur, inscriptisque visceribus hominis incremento pigmenti notae crescunt: nec quicquam mage patientiae loco nationes ferae ducunt, quam ut per memores cicatrices plurimum fuci artus bibant ${ }^{111}$.

La più occidentale delle isole celtiche, così, diviene perenne e vivificante simbolo di una soglia, di un limen, o, ancor meglio, di un varco all'Atlantico settentrionale proprio per quella favolosa e atopica Thule che fu l'ultimo baluardo metastorico e trascendente nell'Oceano. E sarà nelle parole di Patrizio, di un celta romano e cristiano che giunse usque ad ultimum terrae (Confessio 1, 13), che la visione e la stessa realtà di un'Irlanda quale terra liminare ed escatologica, perché già terra incognita, troveranno la loro più bella e concreta definizione, parole che consegnano alla storia religiosa la viva immagine di una Romanitas che attraverso il legno della Croce si

${ }^{110}$ Identificabile con il già ricordato piccolo arcipelago delle Scilly, a circa quaranta chilometri dal promontorio di Land's End, in Cornovaglia. Vedi Dion, 1952.

${ }^{111}$ Collectanea rerum memorabilium 22, ed. T. Mommsen, Berlin 1895, rist. 1958, pp. 99-103. Si veda Hofeneder, 2008. 
innesta su radici pagane in un reciproco scambio di quella eterna linfa che qui, grazie all'ipostasi di un' insula sacra e di un alter orbis, rifulge miticamente sin dal «tempo favoloso delle origini $»^{112}$ nell'epifania terrena della Tradizione sacra: et ubique pergebam causa vestra in multis periculis etiam usque ad exteras partes, ubi nemo ultra erat et ubi numquam aliquis pervenerat qui baptizaret aut clericos ordinaret aut populum consummaret (Confessio 51, 2-5).

Tale concetto liminare si fa ancor più valido per un'isola la cui configurazione fisica ha contribuito a determinarne la singolarità del percorso storico, in un certo senso a sé stante rispetto a quello del resto d'Europa. In questa reductio più d'altre ritornano, per concludere, le parole di Roger Chauviré, poeta e storico che all'Irlanda dedicò quasi una vita («la géographie physique préfigure la géographie humaine et l'histoire» $\left.{ }^{113}\right)$ e, ancor più, di Georges Dumézil, secondo il quale gli antichi Irlandesi vedevano antonomasticamente nel mare che si distendeva ad occidente della loro isola l'espressione mitopoietica e la funzione escatologica di «antichambre de l'au-delà»"14.

RECIBIDO: octubre 2019; ACEPTADO: noviembre 2019.

\section{BIBLIOGRAFIA (studi)}

AlCOCK, L. (1971): Arthur's Britain: History and Archaeology Ad 367-634, London-New York.

Anderson, M. O. (1982): "Dalriada and the Creation of the Kingdom of the Scots", D. WhiTelock R. MCKITTERICK - D. N. DumviLle (edd.): Ireland in early medieval Europe: Studies in Memory of Kathleen Hughes, Cambridge, pp. 106-132.

ANTONELLI, L. (1998): Il periplo nascosto. Lettura stratigrafica e commento storico-archeologico dell Ora Maritima di Avieno, Padova.

Arnold, C. J. (1984): Roman Britain to Anglo-Saxon England, Bloomington.

BATESON, J. D. (1973): “Roman Material from Ireland: A Reconsideration”, Proceedings of the Royal Irish Academy 73C: 21-97.

BATESON, J. D. (1976): "Futher Finds of Roman Material from Ireland", Proceedings of the Royal Irish Academy 76C: 171-180.

BIELeR, L. (1951): "Libri Epistolarum sancti Patricii Episcopi: II. Commentary”, Classica et Mediaevalia 12: 79-214.

Bisagni, J. (2011): "A note on the end of the world: Tírechán’s dies erdathe", Zeitschrift für Celtische Philologie 58: 9-18.

\footnotetext{
${ }^{112}$ Eliade, 1983: 27.

${ }^{113}$ Chauviré, 1949: 5.

${ }^{114}$ Dumézil, 1970: 185.
} 
BORCA, F. (2000): Terra mari cincta. Insularità e cultura romana, Roma.

Burns, C. (1991): “Da Agostino di Canterbury a Enrico VIII”, A. CAPRIOli - L. VACCARO (edd.): Storia Religiosa dell'Inghilterra, Milano, pp. 69-131.

BYrne, F. J. (1973): Irish Kings and High-Kings, London.

CAREY, J. (1996): Saint Patrick, the Druids, and the End of the World, "History of Religions» 36/1: 42-53.

Chauviré, R. (1949): Histoire de l'Irlande, Paris.

Collins, R. - Gerrard, J. (edd.) (2004): Debating Late Antiquity in Britain AD 300-700, Oxford.

Cunliffe, B. (1997): The Ancient Celts, Oxford, repr. Harmondsworth, 1999.

DE ANNA, L. G. (1998): Thule. Le fonti e le tradizioni, Rimini.

de Bernardo Stempel, P. (1999): Nominale Wortbildung des alteren Irischen: Stammbildung und Derivation, Tübingen.

DE VRIES, J. (1991): I Celti, trad. it. (Keltische Religion, Stuttgart 1961), Milano.

Dillon, M. - Chadwick, N. K. (1968): I Regni dei Celti, trad. it. (The Celtic Realms, London 1967), Milano.

Dion, R. (1952): “Le problème des Cassiterides", Latomus 11: 306-314.

DumézIL, G. (1970): Du mythe au roman. La Saga de Hadingus (Saxo Grammaticus, I, v-viii) et autres essais, Paris.

Dumville, D. N. (1972-1974): "Some Aspects of the Chronology of the Historia Brittonum", Bulletin of the Board of Celtic Studies 25: 439-445.

Dumville, D. N. (1975-1976): "Nennius and the Historia Brittonum", Studia Celtica 10-11: 78-95.

Dumville, D. N. (1977): "Sub-Roman Britain: History and Legend", History 62: 173-192.

Eliade, M. (1983): Mito e realtà, trad. it. (Myth and Reality, New York, 1963), Roma.

Foster, S. M. (2004): Picts, Gaels and Scots: Early Historic Scotland, London.

FreEman, P. (2001): Ireland and the Classical World, Austin.

Frere, S. S. (1967): Britannia: A History of Roman Britain, Cambridge.

GonZÁlez Marrero, J. A. (2010): "Las islas atlánticas en el Liber de mensura Orbis terrae del monje geógrafo irlandés Dicuil del siglo IX", Anuario de Estudios Atlánticos 56: 71-90.

Gougaud, L. (1907): "Le noms anciens des Iles Britanniques", Revue des Questions Historiques 83: 537-547.

Haverfield, F. J. (1913): “Ancient Rome and Ireland”, English Historical Review 28: 1-12.

HenNig, R. (1952): “Die britischen Inseln im Altertum”, Saeculum 3: 56-69.

Herren, M. W. - Brown, S. A. (2002): Christ in Celtic Christianity. Britain and Ireland from the fifth to the tenth Century, Woodbridge.

Higham, N. J. (1994): The English Conquest: Gildas and Britain in the 5th Century, Manchester.

Hofeneder, A. (2008): “C. Iulius Solinus als Quelle für die keltische Religion”, A. SARTORI (ed.): Dedicanti e cultores nelle religioni celtiche, Milano, pp. 135-165.

Hogan, E. (1910): Onomasticon Goedelicum locorum et tribuum Hiberniae et Scottiae, Dublin-London.

Holder, A. (1896-1897): Alt-Celtischer Sprachschatz, Leipzig.

Hubert, H. (1932): Les Celtes et l'expansion celtique jusqu'à l'époque de la Tène, Paris.

IANNELlo, F. (2011): "Il processo di cristianizzazione dell'aldilà celtico e delle divinità marine irlandesi nella Navigatio sancti Brendani", 'Ilu. Revista de Ciencias de las Religiones 16: 127-151. 
IANNELlo, F. (2013a): Jasconius rivelato. Studio comparativo del simbolismo religioso dell'isola-balena" nella Navigatio sancti Brendani, Alessandria.

IANNello, F. (2013b): "Notes and Considerations on the Importance of St. Patrick's Epistola ad Milites Corotici as a Source on the Origins of Celtic Christianity and Sub-Roman Britain", Imago Temporis. Medium Aevum 7: 97-137.

Jones, M. E. (1998): The End of Roman Britain, Ithaca-London.

KENNEY, J. F. (1929): The Sources for the Early History of Ireland. Ecclesiastical. An Introduction and Guide, Dublin, repr. with addenda by L. Bieler, New York 1966).

Koch, J. T. (1990): "New Thoughts on Albion, Iernè, and the Pretanic Isles", Proceedings of the Harvard Celtic Colloquium 6: 1-28.

LAING, L. - LAING, J. (1986): "Scottish and Irish metalwork and the conspiratio barbarica", Proceedings of the Society of Antiquaries of Scotland 116: 211-221.

Le Roux, F. (1968): "La mythologie irlandaise du Livre des Conquêtes”, Ogam 20: 381-404.

Le Roux, F. - GuyonvarC'H, C.-J. (1986): Les Druides, Rennes.

Le Roux, F. M. (1962): "Les Iles au Nord du Monde”, N. Renard (ed.): Hommages a Albert Grenier, Bruxelles 1962, pp. 1051-1062.

Levalois, C. (1988): La Terra di Luce. Il Nord e l'Origine, trad. it. (La Terre de lumière: le Nord et l'Origine, Bordeaux 1985), Saluzzo.

López SACO, J. O. (1994): "La muerte y utopía de las Islas de los Bienaventurados en el imaginario griego”, Fortunatae 6: 43-70.

Lотн, J. (1920-1921): “La première apparition des Celtes dans l'Ile de Bretagne et en Gaule”, Revue Celtique 38: 259-288.

LUISELLI, B. (1992): Storia culturale dei rapporti tra mondo romano e mondo germanico, Roma.

LUISELLI, B. (2003): La formazione della cultura europea occidentale, Roma.

Mac CANA, P. (1955-1956): "Aspects of the Theme of King and Goddess in Irish Literaure", Études Celtiques 8: 76-114.

MacKie, E. W. - MacKie, R. M. (1984): "Red-haired “Celts" are better termed Caledonians”, American Journal of Dermatopathology 6 (suppl. 1): 147-149.

MacNeill, E. (1920): Phases of Irish History, Dublin.

Magnani, S. (2002): Il viaggio di Pitea sull'oceano, Bologna.

Malaspina, E. (1984): Patrizio e l'acculturazione latina dell'Irlanda, L'Aquila-Roma.

Malaspina. E. (1985): “Agli albori della cultura latina in Irlanda”, Studi Romani 33: 1-10.

Malaspina, E. (ed.) (1985): Scritti di san Patrizio. Alle origini del cristianesimo irlandese, Roma.

Markale, J. (2001): I Celti, trad. it. (Les Celtes et la civilisation celtique, Paris 1969), Milano.

Maya GonzÁlez, J. L. (1999): Celti e Iberi, Milano.

McNeill, J. T. (1974): The Celtic Churches. A History A.D. 200 to 1200, Chicago-London.

Miller, M. (1982): "Matriliny by Treaty: the Pictish Foundation-Legend”, D. WHITELOCK- R. MCKITTERICK - D. Dumville (edd.): Ireland in Early Medieval Europe. Studies in Memory of Kathleen Hughes, Cambridge, pp. 133-161. 
Morris, J. (1973): The Age of Arthur. A History of the British Isles from 350 to 650, London.

Ó Corrárn, D. (2010): The Church and Secular Society, Aa. Vv., L'Irlanda e gli Irlandesi nell'Alto Medioevo, Settimana di Studio della Fondazione Centro Italiano di Studi sull'Alto Medioevo 57, pp. 261-321.

O'RahilLy, T. F. (1946): Early Irish History and Mythology, Dublin.

Olmsted, G. S. (1982): “Morrigan's warning to Donn Cuailnge”, Études Celtiques 19: 165-172.

POKORNY, J. (1959): Indogermanisches Etymologisches Worterbuch (2 voll.), Bern.

Powell T. G. E. (1959): I Celti, trad. it., Milano, trad. it. (New York 1958).

Rance, P. (2001): "Attacotti, Déisi and Magnus Maximus: the Case for Irish Federates in Late Roman Britain”, Britannia 32: 243-270.

RanKIN, D. (1987): Celts and the Classical World, London-New York.

Rankin, D. (1995): “The Celts through Classical Eyes”, M. J. Green (ed.): The Celtic World, LondonNew York, pp 21-33.

ReEs, A. - ReEs, B. (1961): Celtic Heritage, London.

SANTARCANGeli, P. (1975): "Le isole dei morti”, Conoscenza religiosa 2: 140-163.

SANTORO, V. (1991): "Sul concetto di Britannia tra Antichità e Medioevo", Romanobarbarica 11:321-334.

SERGI, G. (1987): I Britanni, Roma (1 ed. Milano, 1936).

Sims-Williams, P. (1993): “Le lingue celtiche”, A. Giacalone Ramat - P. RAmat (edd.): Le lingue indoeuropee, Bologna, pp. 373-408.

SMith, W. (ed.) (1875): Dictionary of Greek and Roman Antiquities, London.

SMYTH, A. P. (1984): Warlords and Holy Men. Scotland AD 80-1000, London.

SNYDER C. A. (1998): An Age of Tyrants: Britain and the Britons, AD 400-600, University Park-Gloucester.

Sutherland, E. (1994): In search of the Picts: A Celtic Dark Age Nation, London.

Thomas, C. (1981): Christianity in Roman Britain to AD 500, London.

Thomas, C. (1986): Celtic Britain, London.

Tierney, J. J. (1976): “The Greek Geographic Tradition and Ptolomy's Evidence for Irish Geography”, Proceedings of the Royal Irish Academy 76: 257-265.

TOMmasi Moreschini, C. O. (2017): ‘Through Others' Eyes: Reconstructing the Celtic Otherworld from Classical and Late Antique Literary Sources", I. TANASEANU-DÖBLER - A. LeFTERATOU - G. RYSER - K. STAMATOPOUlos (edd.): Reading the Way to the Netherworld. Education and Representations of the Beyond in Later Antiquity, Göttingen-Bristol, pp. 327-351.

Vendryes, J. (1974): Lexique étymologique de l'irlandais ancien. Lettres RS, Dublin-Paris.

Wainwright, F. T. (1958): The Problem of the Picts, Edinburgh.

Whitelock, D. - McKitterick, R. - Dumville, D. (edd.) (1982): Ireland in Early Medieval Europe. Studies in Memory of Kathleen Hughes, Cambridge.

Wright, N. (1984): “Gildas's Geographical Perspective: Some Problems”, M. LAPIDGE - D. N. DumVILle (edd.): Gildas: New Approaches, Cambridge, pp. 85-106.

ZWICKER, J. (1934): Fontes historiae religionis Celticae, vol. 1, Berlin. 
